\title{
Sustainable Choices for Cloud Applications: a Focus on $\mathrm{CO}_{2}$ Emissions
}

\author{
Cinzia Cappiello, Paco Melià, Barbara Pernici, Pierluigi Plebani, Monica Vitali \\ Dipartimento di Elettronica, Informazione e Bioingegneria \\ Politecnico di Milano \\ Piazza Leonardo da Vinci 32, 20133 Milano \\ Email: [name.surname]@polimi.it
}

\begin{abstract}
The widespread adoption of cloud computing is having a big impact on the environment since the energy consumption of data centers and the resulting emissions are significantly increasing. Researchers and practitioners in this field are looking for methods to improve the energy efficiency of data centers and increase the use of green energy sources. In fact, besides the energy consumption, the greenness of a data center can be characterized by the quantity of $\mathrm{CO}_{2}$ emissions associated with the use of electricity (from a specific energy mix) and/or fuels (e.g., for heating or cooling). In this paper, we propose an approach in which environmental impacts are considered as an important factor for the selection of the cloud site for the deployment of applications. In detail, considering a user perspective and focusing on the assessment of energy consumption and $\mathrm{CO}_{2}$ emissions, this paper proposes a method to support the users towards greener choices in the deployment of cloud applications.
\end{abstract}

Index Terms-Cloud applications, $\mathrm{CO}_{2}$ emissions, energy mix, energy consumption.

\section{INTRODUCTION}

Several goals can act as a motivation for making an organization more sustainable. So far, a relevant role has been held by governmental regulations, which put constraints over the amount of $\mathrm{CO}_{2}$ emissions that are allowed for an organization. Another relevant driver is the continuous increasing attention of customers towards sustainability, that has brought customers to prefer companies which demonstrate to care about environmental issues. In this scenario, an increasing number of companies is becoming more and more sensitive to consider the environmental impact of their systems. This happens also for IT companies, especially since data centers and IT systems have a relevant impact on $\mathrm{CO}_{2}$ emissions. Indeed, a report recently produced by Greenpeace [1] assigns to the IT sector the responsibility for the $2 \%$ of the global greenhouse gas (GHG) emissions, pointing out that this percentage is growing. In [2], authors cite data obtained from the US Environmental Protection Agency (EPA), stating that GHG emissions have increased from $4.28 \cdot 10^{13} \mathrm{gCO}_{2}$ in 2007 to $6.79 \cdot 10^{13} \mathrm{gCO}_{2}$ in 2011.

Current trends in research have been often limited to finding methods to reduce the energy demand of data centers. Actually, this is not the only aspect to be considered. As highlighted in the Greenpeace report, an important factor that companies have to consider to move towards a more sustainable asset is the energy production mix (a.k.a. energy mix): it specifies the sources (and their relative importance) used to produce the energy consumed by the cloud facilities (e.g., $80 \%$ coal, $10 \%$ wind, 5\% solar, 5\% nuclear). In fact, energy sources can not be considered as equivalent, because their environmental impacts (e.g., in terms of $\mathrm{CO}_{2}$ emissions) are different. For example, in [3], the authors stress this concept by making a distinction between green and non green energy, and by arguing that the impact due to energy production has to be considered together with the amount of energy consumed.

Focusing on IT solutions, this difference between green and non-green energy sources must be taken into account, especially in data centers, as it might affect the allocation among servers in cloud facilities. In fact, the greenness of a data center, defined as the quantity of $\mathrm{CO}_{2}$ emissions associated with the use of electricity (from a specific energy mix) and/or fuels (e.g. for heating or cooling), can be considered as one of the main drivers for the selection of the cloud site for the deployment of applications.

In this respect, the goal of this paper is to propose an approach for a greener deployment of cloud applications, based on the estimation of energy consumption and $\mathrm{CO}_{2}$ emissions related to these applications. As argued in the rest of the paper, the analysis of the energy mix and the existence of recurring patterns in $\mathrm{CO}_{2}$ emissions at the national level can be exploited to implement a greener deployment model.

The paper is structured as follows. Section II analyzes previous contributions in order to highlight the novelty of the proposed approach. Such approach is described in Section V and it has been designed considering the reference architecture described in Section III. Section IV defines the assessment methods used to calculate the energy mix in the different cloud sites. Finally, Section VI shows, by using several examples, the effectiveness of the proposed approach. Discussion on future directions of this research is given in Section VII that concludes the work.

\section{RELATED WORK}

In a cloud environment, several solutions can be adopted to make IT systems more sustainable. Several researchers focused on finding methods to use available renewable resources in a more efficient way. It is well known that the main issue related to renewable energy sources is that they are not constantly 


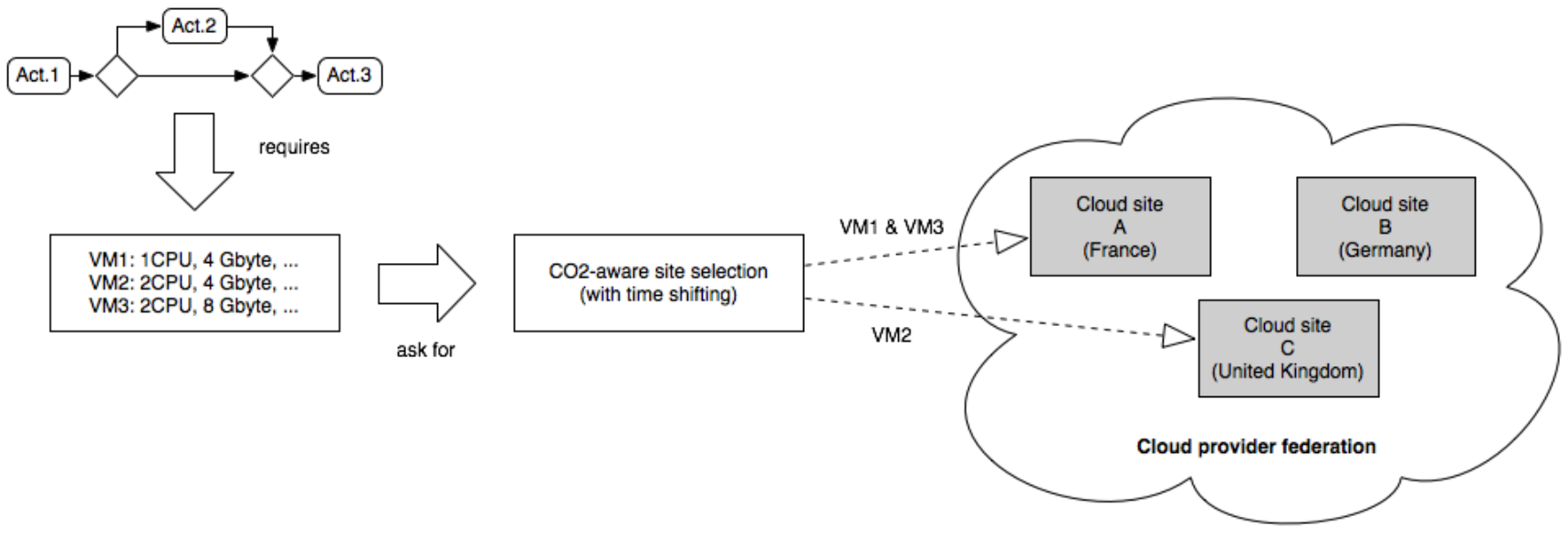

Fig. 1. Reference architecture and scenario

available: their productivity depends on a set of almost unpredictable factors. In [4], authors propose to use a Geographical Load Balancing (GLB) to shift workloads and avoid peak power demand. In order to reach their goals, they propose a prediction algorithm to anticipate future peak demands. The approach is effective for short time predictions, since the error of the algorithm increases with the length of the prediction window. In deciding the workload allocation, the algorithm takes into consideration temporal patterns of generation from renewable energy sources, energy storage devices, and power state of servers. A similar approach is described in [5] in which authors design an algorithm able to reduce the cost due to energy demand for data centers participating in Coincident Peak Pricing (CPP) programs. These programs charge more when the whole network is in peak of requests. In order to avoid peak load demand, the authors use a combination of two techniques: local power generation usage and workload shifting. The algorithm tries to optimize local generation and workload shifting for reducing energy costs. Also in [6], authors highlight the importance of the contribution of green energy sources to promote sustainability in IT systems and propose a load balancing algorithm that takes into account this information in order to decide where to allocate the request.

Some scholars addressed the problem of designing sustainable business processes by focusing their attention on business process reconfiguration. In [7], business processes are redesigned choosing from a set of equivalent fragments for each task. The decision is based on qualitative and quantitative metrics, such as $\mathrm{CO}_{2}$ emissions, air quality, and damages to fauna and flora. A similar approach is used in [8], where some patterns are defined to design green business processes in a cloud environment. Nine different design patterns are described, which explore different ways to modify the business process toward a greener dimension. As an example, alternative processes can be selected on the basis of their sustainability, some compensation activity can be performed to reduce the environmental effects, or migration can be performed to obtain a greener configuration.

An overview about improving the sustainability of a cloud environment is given in [9], where the software development life cycle in a cloud environment is analyzed from a green perspective. Here, the authors propose a framework in which they identify opportunities for energy efficiency in efficient hardware and software selection, network optimization, scheduling and management of VMs, selection of green energy sources, and efficient data center design.

\section{REFERENCE SCENARIO}

The proliferation of cloud platforms has made available to the application developers several infrastructures where to create and deploy their own products. In particular, in this paper, we focus on the IaaS (Infrastructure as a Service) provisioning model, i.e., we assume that the software developers are interested on reserving VMs where the developed application will be executed. Moreover, as a reference scenario we assume that the application that has to be considered is a High Performance Computing (HPC) application. This application can require a set of VMs with different characteristics in terms of number of CPUs, amount of memory, and so on.

With this focus, Fig. 1 gives an overview of the reference architecture and scenario that is considered in this paper. First of all, we are not interested on a single cloud provider but on a federation of cloud providers that can have facilities in different countries. As also discussed in the next section, considering different countries also means considering a different impact on the $\mathrm{CO}_{2}$ emissions. Indeed, these emissions depend on how much green is the energy production that is used by the application and this, in turn, depends on the energy mix. For the sake of simplicity, to compute the greenness of the energy production in this paper we will refer on the energy mix at national level. For this reason, without loss of generality, we do not consider possible situations in which the cloud facilities have their own autonomous power plants.

Having this cloud infrastructure and this kind of applications, the goal of our approach is to find the optimal site 
where to deploy the requested resources. With respect to the state of the art, this site selection does not only consider the usual constraints on the availability of resources that should be compatible to what it is required but it is also aware of the environmental impact, measured in terms of $\mathrm{CO}_{2}$ emissions. Goal of this paper is to focus on this latter aspect analyzing the way in which the energy mix influences this decision in terms of where and when to deploy the application. In particular, we assume that our approach could support the manager of a federated cloud, as it is the responsible of identifying the best site where to deploy the application when requested by the final users.

As shown in Fig. 1, the result of the work done by our approach will be the identification of the best way to assign the execution of a task to a given cloud site. For instance, in our example, we obtain that the cloud provider suggests to run the VMI and VM3 in France, while the VM2 in UK. The three countries included in this example refers to the countries where the three data centers adopted in the ECO2Clouds ${ }^{1}$ project are located.

It is worth noting, that even if we are referring in this paper on HPC-like applications, the approach presented hereafter also works for any other kind of applications. We decided to use HPC application as their execution is limited on time and a proposed approach that implies a delay on the execution has more sense. Nevertheless, the problem of identify the best site remains relevant also for other classes of applications. For instance, in long running (web) applications, in which redeploy can be necessary in case of overload or inefficiencies, our approach can be useful to select the best site where to redeploy a VM.

\section{ENERGyMiX ANALYSIS}

Greener choices in the deployment of cloud applications should be driven by energy consumption and $\mathrm{CO}_{2}$ emissions. This means that users should select the cloud site to deploy their applications by considering not only performance but also green aspects. This requires to collect performance metrics, energy metrics and details about the utilization of green sources.

In our approach, the evaluation of the $\mathrm{CO}_{2}$ emissions is based on the emission factors $(\mathrm{gCO} 2 \mathrm{e} / \mathrm{kWh})$ provided by the national grids. Indeed, given the amount of energy consumed, with this factor is possible to compute the amount of $\mathrm{CO}_{2}$.

Emission factors largely vary from country to country. For example, the three data centers involved in the $\mathrm{ECO}_{2} \mathrm{Clouds}$ project are located in France, Germany and United Kingdom, respectively. Some technical reports describe that the country with the lowest carbon intensity is France, whose power generation is mainly based on nuclear plants. Estimated emission factors for France range between 62 [10] and 146 [11] gCO2e/kWh. In contrast, German energy is more carbonintensive, with emission factor estimates ranging between 629 [12] and 706 [11] gCO2e/kWh. Finally, emission factors for

\footnotetext{
${ }^{1}$ http://www.eco2clouds.eu
}

the United Kingdom are estimated to range between 567 [13] and 658 [11] gCO2e/kWh.

As our goal is to deploy an application in a federated cloud environment, the evaluation and estimation of the emission factors is a very important step in our approach. As the factor may vary over time, it is important to know which is the value of the emission factor when the application will run, so that the optimal deployment can occur.

As a first contribution of this paper, we propose different ways to assess the $\mathrm{CO}_{2}$ emissions.

Looking at the data cited in the previous paragraph, we can observe that there are public documents that periodically publish the average emission factors of the different countries in a specific period. In this case, assuming that we know the average power consumption (AP) for a specific site, the energy $(\mathrm{kWh})$ consumed in a specific period can be calculated by multiplying AP by the number of hours in the considered period. $\mathrm{CO}_{2}$ emissions result multiplying the energy consumed by the emission factor (that is a constant). The AP can be estimated knowing features of the hosts (idle and peak power consumption) and of the application (average resources demand).

Besides the average emission factors, some countries publish the real time energy mix via public web sites. In this case, the assessment and estimation of $\mathrm{CO}_{2}$ emissions could be more comprehensive and meaningful. For example, for two of the three countries that we consider in the $\mathrm{ECO}_{2}$ Clouds project real-time energy mixes are available. In particular, France energy mix can be retrieved through the information service éCO2mix available on the RTE website ${ }^{2}$. Such service shows electricity demand, electricity generation classified by source and cross-border commercial exchanges (imports/exports). Data are update automatically every 15 minutes. Similar information is available for UK. Real time and historic data about the energy generation in UK are available through the BMRS (Balancing Mechanism Reporting System) website ${ }^{3}$. For this web site data are updated every 5 minutes.

The availability of historical data can be exploited in order to identify regular and/or seasonal patterns that can be used in the deployment of applications. For example, a preliminary analysis of french data of January 2012 revealed the presence of a regular pattern of the emission factors during the week days and another pattern for the weekend days (see Figure 3).

The regularity of the emission factors that characterizes the weekdays has been proven by calculating the correlation indexes among the assessed values of the different week days. As shown in Table I, the correlation indexes are significantly high and it is possible to state that the values gathered in the different days are positively correlated, and thus characterized, by a very similar trend.

Such trends can be found also in the other months with the difference of seasonal factors. Also the analysis of the British

\footnotetext{
${ }^{2}$ http://www.rte-france.com/fr/

${ }^{3}$ http://www.bmreports.com/
} 


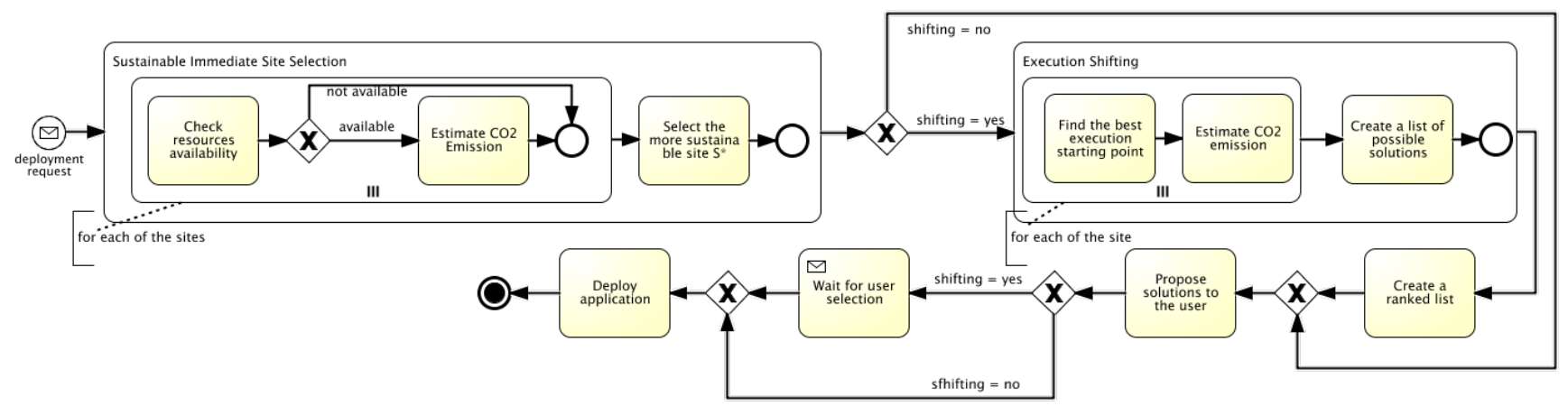

Fig. 2. $\mathrm{CO}_{2}$-driven site selection

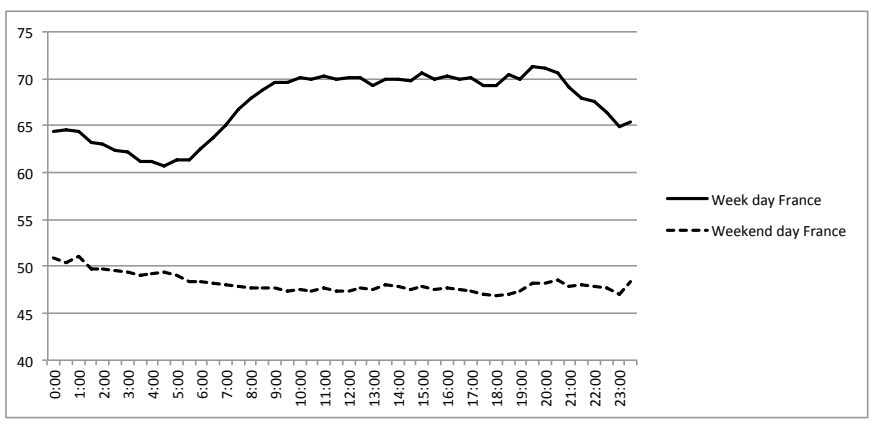

Fig. 3. Trend of the emission factor $(\mathrm{gCO} 2 \mathrm{e} / \mathrm{kWh})$ during week days and week ends in France

TABLE I

CORRELATION INDEXES OF A WEEK MEASUREMENT

\begin{tabular}{|c|c|c|c|c|c|}
\hline & Monday & Tuesday & Wednesday & Thursday & Friday \\
\hline Monday & 1 & 0.92 & 0.67 & 0.75 & 0.69 \\
\hline Tuesday & - & 1 & 0.87 & 0.89 & 0.83 \\
\hline Wednesday & - & - & 1 & 0.91 & 0.92 \\
\hline Thursday & - & - & - & 1 & 0.93 \\
\hline Friday & - & - & - & - & 1 \\
\hline
\end{tabular}

data revealed interesting patterns as better explained in Section VI.

Having these trends, next section will discuss how they can be helpful in driving greener choices for the deployment of cloud applications.

\section{V. $\mathrm{CO}_{2}$-DRIVEN Site Selection}

When an application (e.g. an HPC one) has to be deployed on a cloud environment, the energy mix can be estimated according to what it has been discussed in the previous paragraph for each of the countries where the cloud providers have their facilities. Based on that, this section discusses the second main contribution of the paper: i.e., to provide a more green deployment of the application, by making the user aware of its environmental impact and by allowing him/her to perform some choices that can reduce the carbon footprint of his/her application. In particular, two complementary approaches are proposed:

- Immediate site selection: the approach selects the site according to its carbon footprint, the availability of the resources and the estimated duration of the execution.

- Execution shifting: instead of reserving immediately the requested resources, the system proposes alternative solutions that imply a delay of the reservation. This means that the execution of the application will be delayed as well.

Fig. 2, using BPMN notation, illustrates how these two approaches can take place when the user requests a deployment to the cloud. First of all, the cloud provider enables the user to submit deployment requests for their application. When submitting a request, the user specifies the resources that have to be reserved for the application and an estimated duration of the application (how long the application is going to stay deployed in the cloud infrastructure). The user also specifies his/her availability in postponing the deployment and the acceptable delay.

Given this information, the cloud provider executes the immediate site selection algorithm and selects the site with the lower estimated $\mathrm{CO}_{2}$ consumption for an immediate deployment of the application. Note that such estimation should also take into account potential differences of performance among the considered cloud sites (if the information is available) that can impact on the duration of the application and/or needed power.

In case the user has expressed his/her availability in postponing the execution, the cloud provider performs also the execution shifting algorithm. The output of the algorithm is a list of tuples composed of the name of the site, the delay value, and the estimated $\mathrm{CO}_{2}$ for the solution. Obtained results are compared with the result of the site selection algorithm and ranked according with the estimated $\mathrm{CO}_{2}$ emissions. The cloud provider presents to the user the estimation in case of immediate deployment and then the list of the other solutions, together with the $\mathrm{CO}_{2}$ emission reduction and the delay, with an advice about the most convenient combination. The final 
choice is left to the user.

The two approaches, i.e., immediate and postponed site selection, base their behavior on the patterns retrieved from the analysis of historical values of the $\mathrm{CO}_{2}$ emissions of the considered countries. Details on these approaches follow.

\section{A. Immediate site selection}

This approach consists in selecting the site where to deploy an application based on the estimated current $\mathrm{CO}_{2}$ emissions. Let us consider a general scenario where a cloud infrastructure is distributed on several sites placed in different countries, each one with its own energy mix and $\mathrm{CO}_{2}$ emissions rate. Some countries provide an instantaneous value for $\mathrm{CO}_{2}$ emissions, according to the current production of power. Others provide just a general value that is the average emission value, valid for the whole time. According to this scenario, once a request is received, the infrastructure provider can place the application in the site which is going to be greener than the other ones for that application.

In Sec. IV we have shown that the emissions of $\mathrm{CO}_{2}$ might follow a regular pattern which is dependent on the time of the day and on the day of the week. The knowledge of this information for each of the sites that compose the cloud infrastructure is determinant to predict the amount of $\mathrm{CO}_{2}$ that the request will generate if deployed. The inputs of the immediate site selection algorithm are the application to be deployed, the resources requested by the application, and an estimated duration in time of the application. The algorithm works as follows:

1) Check the availability of the resources requested by the application on each of the sites $S$ available obtaining a subset of sites $S^{\prime}$.

2) For each site in $S^{\prime}$ predict the $\mathrm{CO}_{2}$ emissions due to the execution of the application given its estimated duration:

- If real time values are available then estimate the $\mathrm{CO}_{2}$ emissions considering the patterns detected on the basis of past observations.

- If only a general value is available then just multiply the value for the estimated duration of the application.

3) Compare the estimations and select the site $S^{*}$ with the lowest estimation.

The algorithm for the sustainable immediate site selection can be performed automatically by the cloud provider without involving the user in the decision, since this algorithm just chooses the more sustainable deployment without affecting the performance of the application. On the contrary, the execution shifting algorithm will require the user involvement.

\section{B. Execution shifting}

In case the execution of the application can be postponed in time, other considerations can be made to further reduce the carbon footprint of the application execution.

In this scenario, we assume that, when the deployment request is sent to the cloud infrastructure, the user can leave to the cloud provider the possibility to postpone the application execution on a more efficient period of time. In this case, the system can investigate better allocation that allows a greater reduction in $\mathrm{CO}_{2}$ emissions. The user can also specify the maximum allowed delay for the application execution. The execution shifting algorithm bases its behavior on the knowledge of the $\mathrm{CO}_{2}$ patterns discussed in Sec. IV. Given this knowledge, for each site $S$ where the instantaneous energy mix is known the algorithm works as follows:

1) for each site in $S$ find the best execution starting point given the estimated duration of the application and the maximum allowed delay;

2) for each solution, analyze the resulting $\mathrm{CO}_{2}$ emissions value;

3) propose to the user a list of possible deployment solutions with their associated $\mathrm{CO}_{2}$ emissions estimation.

The users is involved in this process since they can decide which is the best solution for their needs by selecting an option from the ranked list.

\section{VALIDATION}

In this section we use the knowledge acquired from the analysis of patterns in $\mathrm{CO}_{2}$ emissions explained in Sec. IV to validate the algorithm described in Sec. V. Through some examples, we describe the potentiality of the proposed approach in reducing emissions. As before, the algorithm is divided into two steps, the sustainable immediate site selection and the execution shifting, that can be analyzed separately.

\section{A. Sustainable immediate site selection validation}

This part of the algorithm allows the selection of the best site by comparing different outcomes for an immediate deployment of an application on each of them. As explained in Sec. III, in our example we are considering a cloud environment where three sites are available in three different countries: France, Germany, and United Kingdom. From the analysis of the GHG emissions for each of them, we obtained the plots shown in Fig. 3 and in Fig. 4. Each line in the plots represents the average emission factors during a working day and the week end at each of the considered sites. Strong correlations discovered inside a month make us confident that these values can be used as a reference. In the plots the month considered is January 2012, while different results would have been obtained considering other months due to the seasonal variations.

As can be observed, in our scenario the emissions in Germany are represented using a constant value that is equal to $503 \mathrm{gCO} 2 \mathrm{e} / \mathrm{kWh}$. This is due to the fact that the data center has stipulated a contract for the energy provision that guarantees a fix energy mix. For both UK and France, we rely on the real time values available on the Web and a more detailed analysis can be done. However, in this specific case, France has always a lower emission rate than the other two sites due to its extensive use of nuclear plants. According to this, whenever available, the better choice would always be to deploy the application in the data center located in France. 


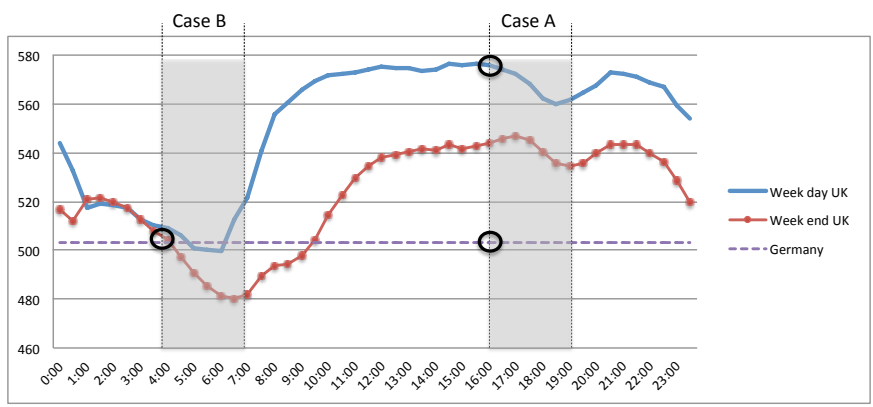

Fig. 4. A detailed view of the trend of emissions factor $(\mathrm{gCO} 2 \mathrm{e} / \mathrm{kWh})$ for Germany and UK

However, resources can also be unavailable for that site, and a comparison is needed for the remaining two sites.

Let us consider a scenario (Case A) where the user asks to deploy an application on Thursday 19th of January at 4:00 p.m. The application requires to be executed for 3 hours and we estimate an energy consumption of $3 \mathrm{kWh}$ (we assume that the considered sites are equivalent from the performance point of view). When the request arrives, resources are available only in UK and Germany, so the set of available sites $S^{\prime}$ is composed of only two sites. A detailed view of the two patterns can be seen in Fig. 4. The estimation for the immediate execution on each site in $S^{\prime}$ results in $1706 \mathrm{gCO} 2 \mathrm{e}$ for UK and $1509 \mathrm{gCO} 2 \mathrm{e}$ for Germany. According to this, the cloud provider decides to deploy the application in Germany. To demonstrate the effectiveness of the decision we have computed which would have been the actual emission of the application using the available data for the considered date. The real consumption in the UK resulted to be $1677 \mathrm{gCO} 2 \mathrm{e}$ and thus the actual saving is $168 \mathrm{gCO} 2 \mathrm{e}$. Let us consider the same scenario but when the request arrives at 4:00 a.m. of Saturday 21th. In this case (Case B), estimated emissions are equal to 1469.5 $\mathrm{gCO} 2 \mathrm{e}$ for UK and $1509 \mathrm{gCO} 2 \mathrm{e}$ for Germany. The best choice consists in deploying the application in UK where with the real consumption of $1349.5 \mathrm{gCO} 2 \mathrm{e}$ it is possible to save 159.5 $\mathrm{gCO} 2 \mathrm{e}$, even if at the time of the request, Germany had a better emission rate.

\section{B. Execution shifting validation}

In this paragraph we validate the second part of the approach, where the customer agrees to postpone the deployment of his application. In order to avoid redundancy we analyze the situation on a single site and for this evaluation we refer to data collected for emissions in France, as shown in Fig. 3. However, the same procedure should be repeated at each site, as discussed in Sec. V-B. Let us consider the same scenario discussed in the previous paragraph where a request arrives on Thursday 19th of January at 4:00 p.m. The user specifies his availability in postponing the execution with a maximum delay of 48 hours. From an analysis of the trend, the execution shifting algorithm proposes several solutions to the user. The first solution consists in the immediate deployment, with an estimated emission of $209.7 \mathrm{gCO} 2 \mathrm{e}$. The second
TABLE II

COMPARISON OF EXECUTION SHIFTING OUTCOMES

\begin{tabular}{|l|c|c|c|c|}
\hline & Delay & Estimated gCO2e & Real gCO2e & Saving (\%) \\
\hline Solution 1 & 0 & 209.7 & 200.3 & - \\
\hline Solution 2 & $10 \mathrm{~h}$ & 185.4 & 167.1 & $16.6 \%$ \\
\hline Solution 3 & $27 \mathrm{~h}$ & 143.2 & 140.3 & $30 \%$ \\
\hline
\end{tabular}

solution consists in delaying the execution of 10 hours, by deploying the application on Friday 20th at 2:00 a.m. In this case, the estimated saving is $24.35 \mathrm{gCO} 2 \mathrm{e}$. The last solution propose the execution in the week end, starting at 7:00 a.m. of Saturday 21st, with a delay of 27 hours and an estimated saving of $66.5 \mathrm{gCO} 2 \mathrm{e}$. The user can decide which solution is better according to his needs. In Tab. II the three solutions are compared. The table reports both the estimated and the real values for $\mathrm{CO}_{2}$ emissions for the three solutions. In the last column it is possible to see the saving in emissions that is obtained when delaying the application deployment. This value is obtained by comparing the effective emissions of the solution to the outcome of the immediate deployment. In this specific example, the algorithm can reduce the emissions of the $30 \%$.

\section{CONCLUding REMARKS}

This paper introduce an approach for considering $\mathrm{CO}_{2}$ emissions as a relevant dimension to be considered when applications have to be deployed in a federated cloud. Based on the experience gained in the $\mathrm{ECO}_{2}$ Clouds project, the paper proposed a method to analyze the energy mix to discover patterns that can be used to optimize the deployment phase. Moreover, the paper introduces a site selection algorithm that considers $\mathrm{CO}_{2}$ emissions in two cases: an immediate deployment and a delayed deployment. A validation scenario based on real data publicly available on the energy mix of France and UK shows how energy savings can be obtained by following appropriate deployment strategies.

Next steps in this research will take into account the impact of different energy sources from cradle to cradle. This means, for instance, that the nuclear power will not be considered as a green energy source. In fact, while $\mathrm{CO}_{2}$ emissions of nuclear power plants are negligible during the operational phase, plant construction and decommissioning after end of life cause considerable impacts, as does the storage and management of nuclear waste. Risks associated to different energy sources is another crucial point that should be considered in future analysis. Users might indeed prefer to avoid choosing cloud sites fed with energy sources perceived as possible causes of severe environmental contamination in case of accident.

\section{ACKNOWLEDGMENT}

This work has been supported by the $\mathrm{ECO}_{2}$ Clouds project (http://eco2clouds.eu/) and has been partly funded by the 
European Commission's IST activity of the 7th Framework Programme under contract number 318048.

\section{REFERENCES}

[1] G. Cook, "How Clean is Your Cloud?," tech. rep., Greenpeace International, April 2012.

[2] A. Beloglazov, R. Buyya, Y. C. Lee, and A. Zomaya, "A taxonomy and survey of energy-efficient data centers and cloud computing systems," Advances in Computers, vol. 82, no. 2, pp. 47-111, 2011.

[3] J.-M. Pierson, "Green Task Allocation: Taking into Account the Ecological Impact of Task Allocation in Clusters and Clouds," Journal of Green Engineering, vol. 1, no. 02, p. 11761, 2011.

[4] Z. Abbasi, M. Pore, and S. Gupta, "Impact of workload and renewable prediction on the value of geographical workload management," in Second International Workshop on Energy Efficient Data Centers (E2DC), 2013.

[5] Z. Liu, A. Wierman, Y. Chen, B. Razon, and N. Chen, "Data center demand response: Avoiding the coincident peak via workload shifting and local generation," Performance Evaluation, vol. 70, no. 10, pp. 770791,2013
[6] Z. Liu, M. Lin, A. Wierman, S. H. Low, and L. L. H. Andrew, "Geographical load balancing with renewables," In Proc. of Sigmetrics $2011,2011$.

[7] K. Hoesch-Klohe and A. Ghose, "Carbon-Aware Business Process Design in Abnoba," in ICSOC, pp. 551-556, Springer, 2010.

[8] A. Nowak, T. Binz, C. Fehling, O. Kopp, F. Leymann, and S. Wagner, "Pattern-driven green adaptation of process-based applications and their runtime infrastructure," Computing, vol. 94(6), pp. 463-487, 2012.

[9] N. S. Chauhan and A. Saxena, "A Green Software Development Life Cycle for Cloud Computing," IT Professional, vol. 15, no. 1, pp. 28-34, 2013.

[10] ADEME, "Guide des facteurs dmissions, Version 6.1. Chapitre 2, Facteurs associs la consommation directe dnergie," tech. rep., 2010.

[11] European Commission, "European Commission (2010) How to develop a Sustainable Energy Action Plan (SEAP)," tech. rep., 2010.

[12] Umweltbundesamt, "Entwicklung der spezifischen KohlendioxidEmissionen des deutschen Strommix 1990-2010 und erste Schtzungen 2011," tech. rep., 2012.

[13] Department for Environment, Food and Rural Affairs, "Guidelines to Defra/DECC's GHG Conversion Factors for Company Reporting: Methodology Paper for Emission Factors," tech. rep., 2012. 